$06,11,13$

\title{
Вынужденный пироэффект в планарном поле
}

\author{
(C) В.Б. Широков ${ }^{1,2}$, А.Г. Разумная ${ }^{2}$, Ю.И. Юзюк $^{2}$ \\ ${ }^{1}$ Южный научный центр РАН, \\ Ростов-на-Дону, Россия \\ 2 Южный федеральный университет, \\ Ростов-на-Дону, Россия \\ E-mail: shirokov-vb@rambler.ru
}

(Поступила в Редакцию 25 октября 2016 г.)

Исследованы действие вынужденной деформации и влияние планарного электрического поля на основное состояние сегнетоэлектрических тонких пленок $\mathrm{Ba}_{0.7} \mathrm{Sr}_{0.3} \mathrm{O}_{3}$, осажденных на (001)-ориентированные кубические подложки. Для значений вынужденной деформации, соответствующих различным основным состояниям, построена зависимость пироэлектрического коэффициента от величины электрического поля $E_{x}$ в плоскости пленки. В областях, где пленка может работать в режиме диэлектрического болометра, наблюдается значение поля, при котором пирокоэффициент достигает экстремального значения. Существует значение вынужденной деформации, при котором пироэффект проявляется наиболее ярко.

Работа выполнена за счет средств Российского научного фонда (грант № 14-12-00258).

DOI: 10.21883/FTT.2017.05.44377.397

\section{1. Введение}

Разработка и совершенствование микроэлектронных устройств опираются на поиск и использование материалов, обладающих уникальными электрофизическими свойствами. Одним из технологических направлений модификации (управления) свойств уже известных нелинейных материалов является тонкопленочная технология. Благодаря значительному деформационному влиянию подложки эта технология дает возможность в широких пределах менять свойства пленок $[1,2]$. Влияние на сегнетоэлектрики электрического поля позволяет создавать элементы электронных устройств, управляемых электрическим полем $[3,4]$.

Сегнетоэлектрические материалы могут обладать хорошими пироэлектрическими свойствами. Применение таких материалов в качестве неохлаждаемых приемников ИК-излучения может быть более эффективным, чем использование других детекторов, из-за их низкого энергопотребления, широкого рабочего диапазона температур и относительно низкой стоимости [5-9]. Если температура ниже точки Кюри, то сегнетоэлектрик всегда обладает пироэффектом. При температурах выше точки Кюри сегнетоэлектрик будет обладать пироэффектом, если к нему приложить постоянное электрическое поле (диэлектрический болометр), причем пирокоэффициент будет зависеть от величины приложенного поля $[5,6]$.

Сегнетоэлектрические твердые растворы титаната бария-стронция $\mathrm{Ba}_{x} \mathrm{Sr}_{1-x} \mathrm{TiO}_{3}$ (BST) при комнатной температуре проявляют уникальные свойства, позволяющие использовать их в качестве управляемых микроэлектронных устройств. Обладая особыми диэлектрическими свойствами и имея низкие диэлектрические потери, твердые растворы BST являются перспективным материалом для применения в микро- и наносистемной технике [10-12]. Для всего ряда твердых растворов построена термодинамическая теория, которая позволяет описывать физические свойства как объемных образцов, так и их тонких пленок $[13,14]$.

В настоящей работе на основе результатов [14] исследуются пироэлектрические свойства тонких пленок твердого раствора $\mathrm{Ba}_{0.7} \mathrm{Sr}_{0.3} \mathrm{TiO}_{3}$ (BST07). Изучены особенности поведения пироэлектрического коэффициента при действии вынужденной деформации (misfitstrain), появляющейся в результате влияния подложки. Также рассмотрено поведение пирокоэффициента под действием электрического поля, направленного вдоль одной из осей в плоскости пленки. Исследование выполнено для монокристаллической монодоменной пленки, осажденной на (001)-ориентированную кубическую подложку. В работе не рассматривается влияние гетероструктуры, т. е. результаты относятся к материалу пленки без учета действия границ.

\section{2. Результаты и обсуждение}

2.1. Основное состояние пленки. Для твердого раствора BST с концентрацией $x=0.7$, как это следует из $[13,14]$, ротационный параметр порядка (ПП), связанный с поворотами октаэдров, можно не учитывать, а ограничиться только ПП-поляризацией. Используя объемный потенциал восьмой степени, термодинамический потенциал для пленки BST07, осажденной на (001)-поверхность кубической подложки, запишем 
в виде [14]

$$
\begin{gathered}
G=a_{1}\left(p_{x}^{2}+p_{y}^{2}\right)+a_{3} p_{z}^{2}+a_{11}\left(p_{x}^{4}+p_{y}^{4}\right)+a_{33} p_{z}^{4} \\
+a_{12} p_{x}^{2} p_{y}^{2}+a_{13}\left(p_{x}^{2}+p_{y}^{2}\right) p_{z}^{2}+G_{6}+G_{8}, \\
G_{6}=a_{111}\left(p_{x}^{6}+p_{y}^{6}+p_{z}^{6}\right)+a_{112}\left[p _ { x } ^ { 4 } \left(p_{y}^{2}\right.\right. \\
\left.\left.+p_{z}^{2}\right)+p_{y}^{4}\left(p_{x}^{2}+p_{z}^{2}\right)+p_{z}^{4}\left(p_{x}^{2}+p_{y}^{2}\right)\right]+a_{123} p_{x}^{2} p_{y}^{2} p_{z}^{2}, \\
G_{8}=a_{111}\left(p_{x}^{8}+p_{y}^{8}+p_{z}^{8}\right)+a_{1112}\left[p_{x}^{6}\left(p_{y}^{2}+p_{z}^{2}\right)\right. \\
\left.+p_{y}^{6}\left(p_{x}^{2}+p_{z}^{2}\right)+p_{z}^{6}\left(p_{x}^{2}+p_{y}^{2}\right)\right]+a_{1122}\left(p_{x}^{4} p_{y}^{4}+p_{x}^{4} p_{z}^{4}\right. \\
\left.+p_{y}^{4} p_{z}^{4}\right)+a_{1123}\left(p_{x}^{4} p_{y}^{2} p_{z}^{2}+p_{x}^{2} p_{y}^{4} p_{z}^{2}+p_{x}^{2} p_{y}^{2} p_{z}^{4}\right),
\end{gathered}
$$

где $p_{i}(i=x, y, z)$ - компоненты вектора поляризации, $a_{i}$ - коэффициенты, численные значения которых для пленки BST с концентрацией $x=0.7$ приведены в таблице.

Основное состояние пленки находится из условия

$$
E_{i}=\frac{\partial G}{\partial p_{i}} .
$$

При комнатной температуре в нулевом внешнем поле в зависимости от величины вынужденной деформации $u_{m}$ пленка может находиться в следующих трех состояниях:

1) $c$-фаза - сегнетоэлектрическое состояние со спонтанной поляризацией, направленной по нормали к плоскости пленки: $p_{x}=0, p_{y}=0, p_{z} \neq 0$ (это состояние реализуется при значениях вынужденной деформации $u_{m} \leq-0.38 \cdot 10^{-3}$;

2) 0-фаза - параэлектрическое состояние с нулевой спонтанной поляризацией (это состояние может реализоваться при значениях деформации, лежащей в пределах $\left.-0.38 \cdot 10^{-3} \leq u_{m} \leq 0.42 \cdot 10^{-3}\right)$;

Значения коэффициентов потенциала феноменологической теории [14] для эпитаксиальной пленки BST $(x=0.7)$ на (001)-срезе кубической подложки при температуре $T=300 \mathrm{~K}\left(u_{m}-\right.$ вынужденная деформация пленки относительно свободного объемного образца)

\begin{tabular}{r|c|c|c}
\hline $\begin{array}{c}\text { № } \\
\text { п/п }\end{array}$ & Коэффициент & Значение & $\begin{array}{c}\text { Единица } \\
\text { измерения }\end{array}$ \\
\hline 1 & $a_{1}$ & $3.8482-9.1744 u_{m} \cdot 10^{3}$ & $10^{6} \mathrm{~J} \cdot m^{5} / C^{4}$ \\
2 & $a_{3}$ & $3.8482+1.0091 u_{m} \cdot 10^{4}$ & \\
3 & $a_{11}$ & 2.0447 & $10^{8} \mathrm{~J} \cdot m^{5} / C^{4}$ \\
4 & $a_{12}$ & 3.5375 & \\
5 & $a_{13}$ & 3.7627 & \\
6 & $a_{33}$ & 0.0802 & $10^{9} \mathrm{~J} \cdot m^{9} / C^{6}$ \\
7 & $a_{111}$ & 0.9058 & \\
8 & $a_{112}$ & -1.3650 & $10^{10} \mathrm{~J} \cdot m^{13} / C^{8}$ \\
9 & $a_{123}$ & -0.5320 & \\
10 & $a_{1111}$ & 2.7041 & \\
11 & $a_{1112}$ & 1.7703 & \\
12 & $a_{1122}$ & 1.1459 &
\end{tabular}

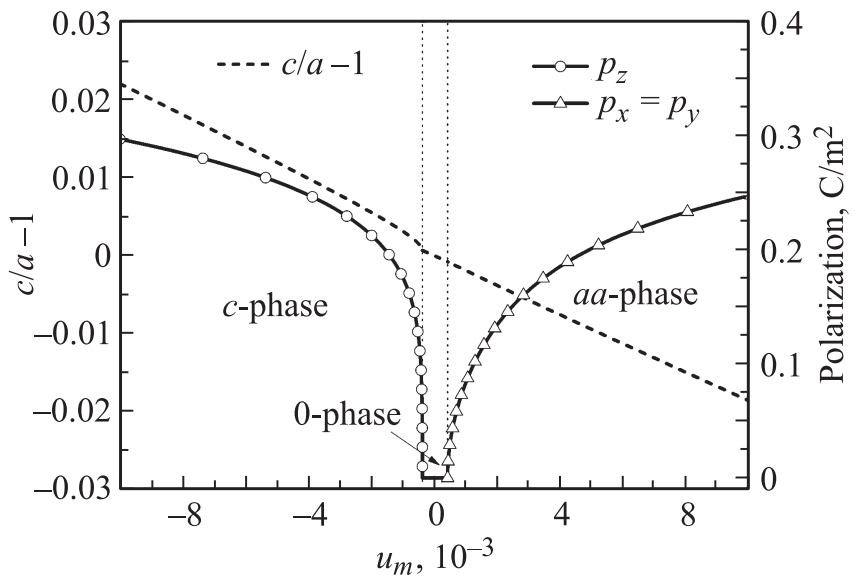

Рис. 1. Зависимость степени тетрагонального искажения $c / a-1$ и значений компонент спонтанной поляризации пленки BST07 от величины вынужденной деформации $u_{m}$.

3) аа-фаза - сегнетоэлектрическое состояние со спонтанной поляризацией, лежащей в плоскости пленки: $p_{x}=p_{y} \neq 0, p_{z}=0$ (это состояние реализуется при значениях вынужденной деформации $u_{m} \geq 0.42 \cdot 10^{-3}$ ).

На рис. 1 показаны зависимости степени тетрагонального искажения $c / a-1$ и значений компонент спонтанной поляризации пленки BST07 от величины вынужденной деформации $u_{m}$. Этот рисунок позволяет оценить величину вынужденной деформации исходя из рентгеноструктурных измерений параметров решетки для конкретной пленки.

2.2. Фазовые переходы в пленке во внешнем поле. При наличии электрического поля $E_{x}$ в плоскости пленки, направленного вдоль одной из кубических осей подложки, симметрийные различия между 0-фазой и $a$-фазой исчезают, так как в этом случае всегда есть ненулевая компонента $p_{x}$, индуцированная приложенным внешним полем. Полевая фазовая диаграмма $\left(E_{x}, u_{m}\right)$ приведена на рис. 2. Граничные точки $T_{1} \quad E_{x}=0 \mathrm{~V} / \mathrm{m}, u_{m}=-0.38 \cdot 10^{-3}$ и $T_{2} \quad E_{x}=0 \mathrm{~V} / \mathrm{m}$, $u_{m}=0.42 \cdot 10^{-3}$ определяют вершины бифуркационных кривых. Слева от точки $T_{1}$ появляется расширяющаяся область существования $a c$-фазы, в которой наряду с индуцированной полем $p_{x}$-компонентной поляризации спонтанно появляется $p_{z}$-компонента. Справа от точки $T_{2}$ появляется расширяющаяся область, где пленка находится в $a b$-фазе. В отсутствие поля это $a a$-фаза с поляризацией, лежащей в плоскости пленки с одинаковыми компонентами $p_{x}=p_{y}$. Переход между $a b$-фазой и $a$-фазой - переход второго рода (штриховая линия на рис. 2). На рис. 2 в области существования $a b$-фазы пунктирной линией показана область переключения $p_{x}$-компоненты поляризации.

На рис. 3, $a-5, a$ приведены зависимости компонент поляризации от планарной компоненты поля $E_{x}$, соответствующие значениям вынужденной деформации для качественно различных сечений фазовой диаграммы, 


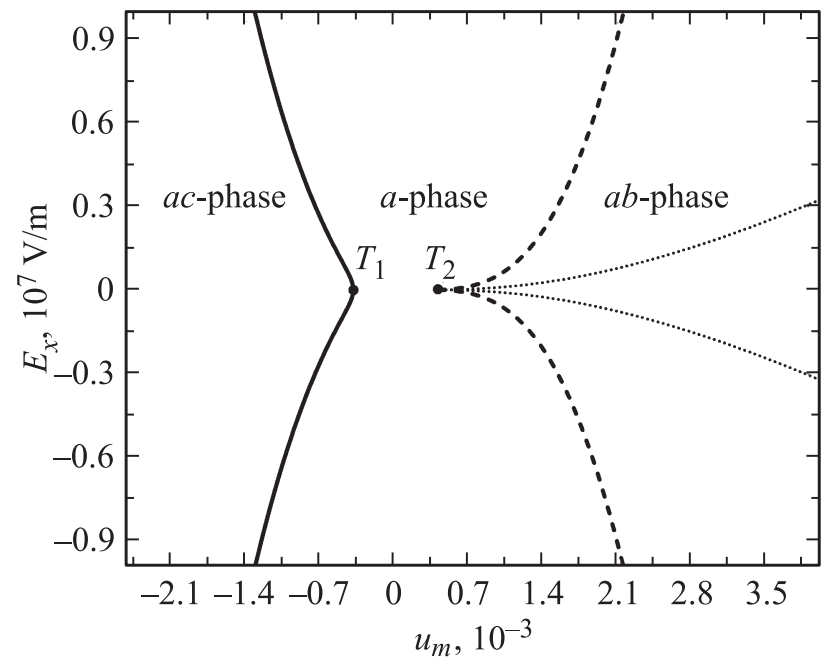

Pис. 2. Фазовая диаграмма тонкой пленки твердого раствора $\mathrm{Ba}_{0.7} \mathrm{Sr}_{0.3} \mathrm{TiO}_{3}$ в координатах поле $E_{x}-$ вынужденная деформация $u_{m}$. Координаты точек $\left(E_{x}, u_{m}\right): T_{1}\left(0,-0.381 \cdot 10^{-3}\right)$, $T_{2}\left(0,0.42 \cdot 10^{-3}\right)$.

приведенной на рис. 2. На рис. 3, $a$ показано поведение компонент поляризации, соответствующее сечению $u_{m}=-1 \cdot 10^{-3}$. Гистерезисные явления здесь связаны с исчезновением компоненты $p_{z}$ при увеличении амплитуды поля $E_{x}$. Качественно такой же вид зависимости компонент поляризации от поля будет для всех сечений левее точки $T_{1}$ при значениях вынужденной деформации $u_{m}<-0.38 \cdot 10^{-3}$ (рис. 2). На рис. 4, $a$ показана зависимость компонент поляризации от поля при $u_{m}=1.0 \cdot 10^{-3}$. Здесь при увеличении поля наблюдаются две особенности. Первая связана с инвертированием компоненты $p_{x}$, при этом вторая компонента $p_{y}$, как это видно из рис. 4, также испытывает скачок. Вторая особенность связана с исчезновением компоненты $p_{y}$, когда пленка переходит в $a$-фазу. Подобная зависимость соответствует сечениям диаграммы правее точки $T_{2}$, т.е. при $u_{m}>0.42 \cdot 10^{-3}$ (рис. 2). На рис. 5, $a$ показана зависимость поляризации от поля при $u_{m}=0$, характерная для параэлектрического состояния. Подобная зависимость будет соответствовать любому сечению диаграммы рис. 2 между точками $T_{1}$ и $T_{2}$ : $-0.38 \cdot 10^{-3}<u_{m}<0.42 \cdot 10^{-3}$.

2.3. Пироэлектрические свойства пленки. Пироэлектрический коэффициент определяется как скорость изменения поляризации при изменении температуры [15]. В том случае, когда существует только одна компонента поляризации, направленная вдоль приложенного поля, пироэлектрический коэффициент вычисляется как

$$
\gamma_{i}=\frac{\partial p_{i}}{\partial T}=-\frac{\frac{\partial^{2} G}{\partial p_{i} \partial T}}{\frac{\partial^{2} G}{\partial p_{i}^{2}}},
$$

с потенциалом $G(1)$, в котором влияние подложки учтено введением параметра вынужденной деформации $u_{m}$.
Индекс $i$ обозначает координату, вдоль которой действует внешнее поле. В рассматриваемом случае $i=x$, т. е. поле направлено вдоль оси $x$ : $E_{x}$. Такое рассмотрение для BST07 возможно только в области существования парафазы.

В параэлектрической фазе пироэлектрический коэффициент при изменении поля имеет максимум. Существование максимума при действии поля - явление универсальное для параэлектрического состояния. Это можно показать в простой модели теории Ландау с потенциалом четвертой степени. Для этого запишем потенциал Ландау в приведенном виде

$$
f=(t-1) p^{2}+p^{4}-E p .
$$

Тогда для пирокоэффициента получим выражение

$$
\gamma=\frac{\partial p}{\partial t}=-\frac{p}{6 p^{2}+t-1} .
$$

С учетом уравнения состояния $\partial f / \partial p=0$ для потенциала (4) получим для точек экстремума пирокоэффициента следующее выражение:

$$
\begin{gathered}
\gamma_{\max }=\mp \frac{\sqrt{6}}{12 \sqrt{t-1}}, \\
E_{\max }= \pm \frac{4 \sqrt{6}}{9}(t-1)^{3 / 2} .
\end{gathered}
$$

Из (5) следует, что экстремум пирокоэффициента существует всегда при $t>1$, т. е. в параэлектрической фазе.

Действие поля $E_{x}$ в $c$-фазе приводит к появлению наряду с компонентой $p_{z}$ и второй компоненты поляризации $p_{x}$. Действие поля $E_{x}$ в $a a$-фазе приводит к тому, что компоненты $p_{x}$ и $p_{y}$ становятся различными. Для состояний с двумя компонентами поляризации пироэлектрический коэффициент равен

$$
\gamma_{x}=\frac{\partial p_{x}}{\partial T}=-\frac{\frac{\partial^{2} G}{\partial p_{i}^{2}} \frac{\partial^{2} G}{\partial p_{x} \partial T}-\frac{\partial^{2} G}{\partial p_{i} \partial p_{x}} \frac{\partial^{2} G}{\partial p_{i} \partial T}}{\frac{\partial^{2} G}{\partial p_{i}^{2}} \frac{\partial^{2} G}{\partial p_{x}^{2}}-\left(\frac{\partial^{2} G}{\partial p_{i} \partial p_{x}}\right)^{2}} .
$$

Здесь индекс $i$ равен $y, z$ для аa-фазы и $c$-фазы соответственно. Формулы (3), (6) учитывают нелинейные уравнения состояния (2) и не предполагают выделения малой добавки к спонтанной поляризации, связанной с откликом на внешнее воздействие, как это принято в [16].

Поведение пироэлектрического коэффициента $\gamma_{x}$ в трех различных областях фазовой диаграммы (рис. 2) показано на рис. $3, b-5, b$. Для различных сечений фазовой диаграммы левее точки $T_{1}$ поведение пироэлектрического коэффициента имеет одинаковый вид. На рис. $3, b$ приведено поведение пирокоэффициента при значении вынужденной деформации $u_{m}=-1 \cdot 10^{-3}$. При изменении поля пирокоэффициент слабо меняется, за исключением области фазового перехода. При достижении границы устойчивости $a c$-фазы пирокоэффициент аномально увеличивается. При обратном движении пирокоэффициент испытывает небольшой скачок. 

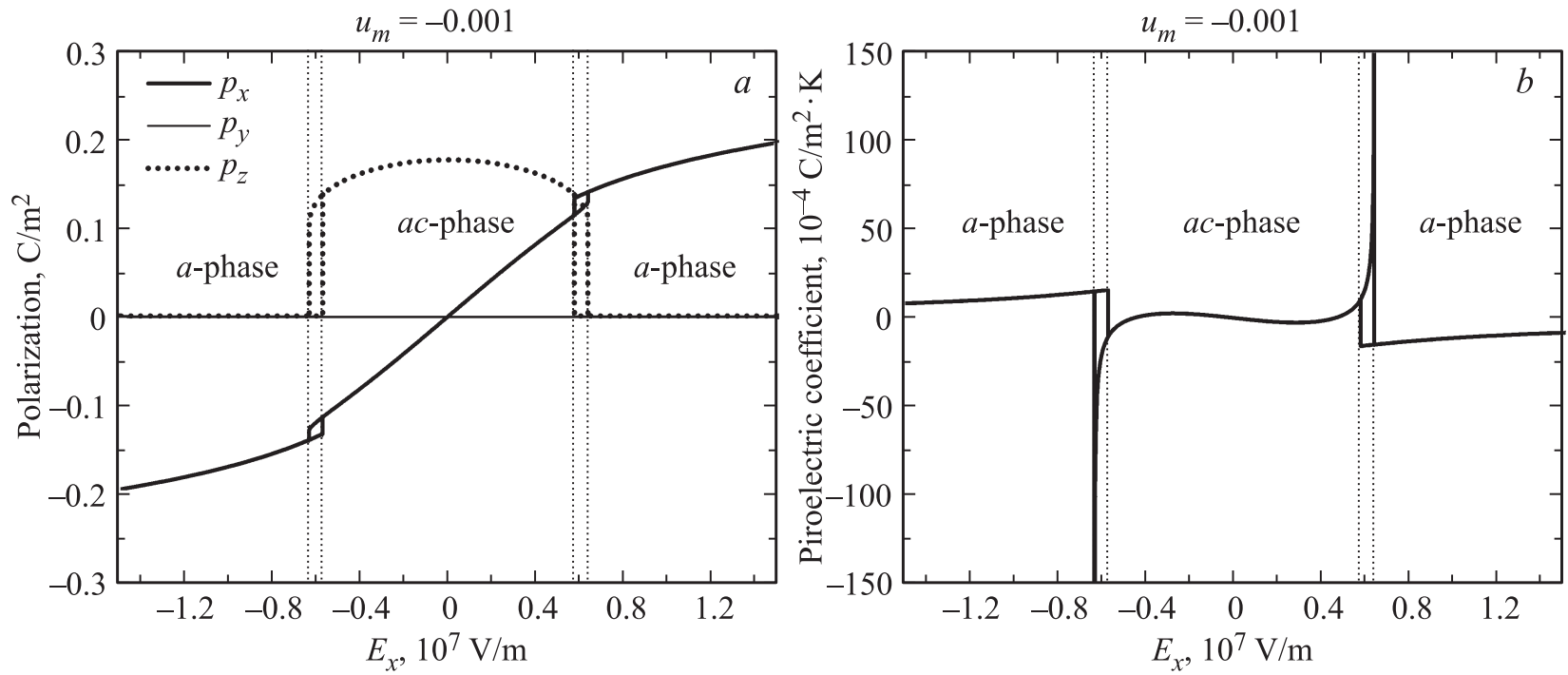

Рис. 3. Поведение поляризации $(a)$ и пироэлектрического коэффициента $(b)$ в зависимости от величины приложенного поля $E_{x}$ при значении вынужденной деформации $u_{m}=-1 \cdot 10^{-3}$. Вертикальные пунктирные линии - границы фаз.
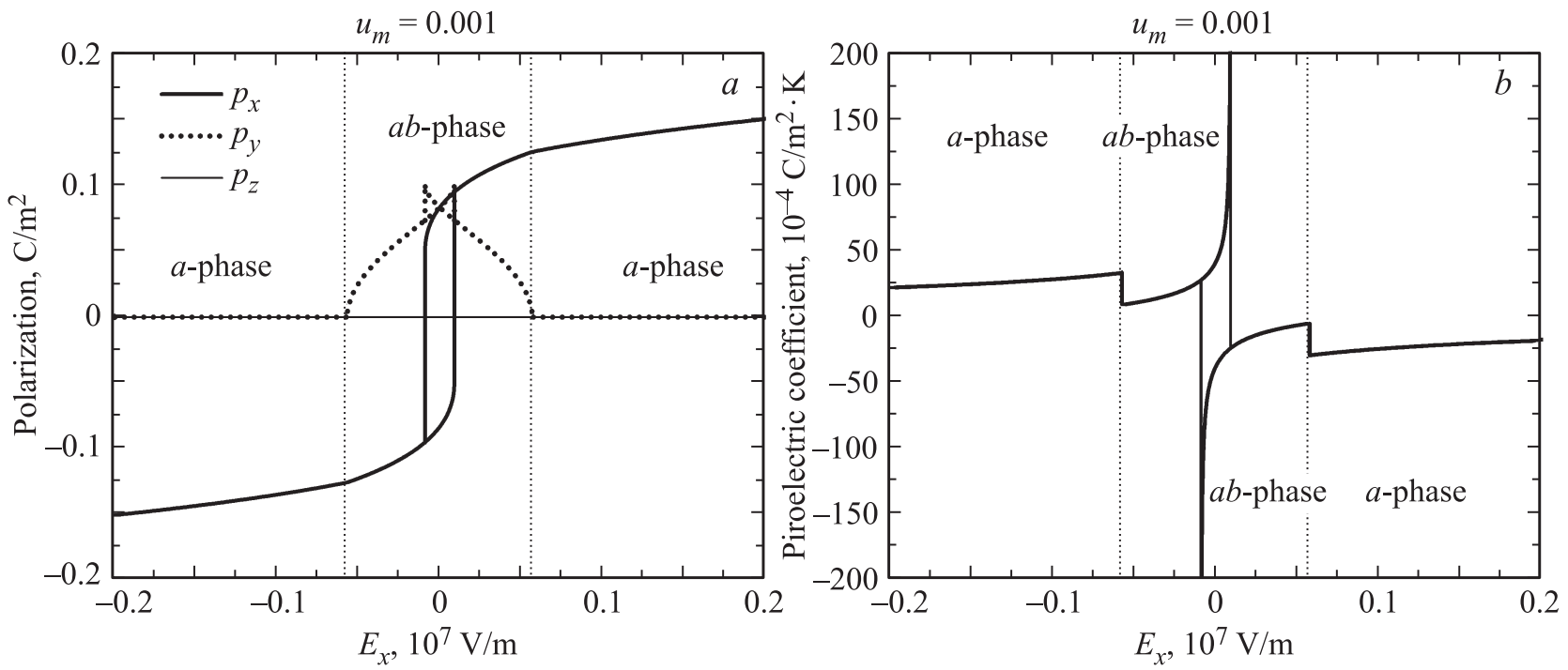

Рис. 4. Поведение поляризации $(a)$ и пироэлектрического коэффициента $(b)$ в зависимости от величины приложенного поля $E_{x}$ при значении вынужденной деформации $u_{m}=1 \cdot 10^{-3}$. Вертикальные пунктирные линии — границы фаз.

При значениях вынужденной деформации правее точки $T_{2}$ в малых полях наблюдается переключение $p_{x}$-компоненты поляризации в $a b$-фазе. Поведение пирокоэффициента для этой области при значении $u_{m}=1 \cdot 10^{-3}$ показано на рис. 4, $b$. Здесь наблюдается аномальное изменение пирокоэффициента при приближении к коэрцитивному значению поля $E_{x}$. Дальнейшее увеличение поля приводит к переходу в $a$-фазу со скачкообразным изменением значения пирокоэффициента.

Основным состоянием пленки BST07 со значением вынужденной деформации $-0.38 \cdot 10^{-3}<u_{m}<0.42 \cdot 10^{-3}$, лежащим между точками $T_{1}$ и $T_{2}$ (рис. 2), является параэлектрическая фаза со спонтанной поляризацией, равной нулю, и, следовательно, равным нулю пироэлектрическим коэффициентом. Под действием поля $E_{x}$ появляется индуцированная поляризация $p_{x}$ и пироэлектрический коэффициент, отличный от нуля. Поведение пирокоэффициента (его абсолютное значение) в этой области значений вынужденной деформацией показано на рис. 5, $b$. Здесь приведены зависимости пирокоэффициента от поля для значений $u_{m}$, лежащих в пределах от $-0.38 \cdot 10^{-3}$ до $0.4 \cdot 10^{-3}$ (с шагом $0.078 \cdot 10^{-3}$ ). Как видно из рис. $5, b$, пирокоэффициент при росте внешнего поля $E_{x}$ начинает увеличиваться от нуля до некоторого максимального значения. По мере дальнейшего увеличения поля пирокоэффициент уменьшается. При приближении величины вынужденной деформации $u_{m}$ к значению вблизи точки $T_{2}$ (рис. 2) абсолютное значение экстремума пирокоэффициента значительно увеличивается. 

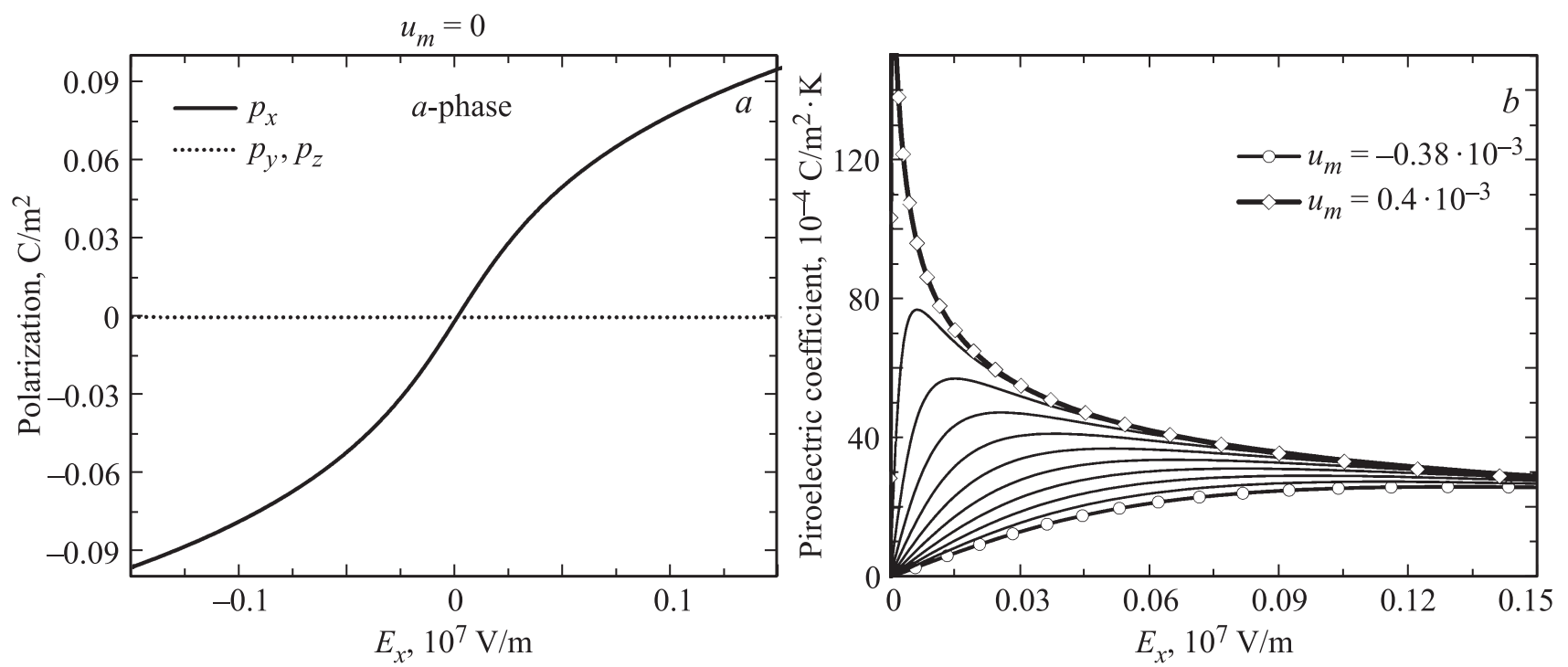

Рис. 5. Поведение поляризации $\left(u_{m}=0\right)(a)$ и абсолютной величины пироэлектрических коэффициентов $(b)$. Линии на части $b$ соответствуют значениям вынужденной деформации $u_{m}$, последовательно меняющейся от $-0.38 \cdot 10^{-3}$ до $0.4 \cdot 10^{-3} \mathrm{c}$ шагом $0.078 \cdot 10^{-3}$.

\section{3. Заключение}

Мы исследовали основное состояние тонкой пленки BST07 при различных значениях вынужденной деформации и приложенного внешнего поля вдоль поверхности пленки. При сжимающих деформациях $\left(u_{m}<0\right)$ пленка может находиться в различных состояниях: в $а с$-фазе в случае малых полей $E_{x}$ и в $a$-фазе при больших полях $E_{x}$. При растягивающих деформациях $\left(u_{m}>0\right)$ пленка может находиться: в $a b$-фазе при малых полях $E_{x}$, и в $a$-фазе при больших. При малых деформациях пленка находится в $a$-фазе.

Пироэлектрический коэффициент ведет себя по-разному в различных областях фазовой диаграммы в зависимости от характера основного состояния. В состояниях с компонентами поляризации, существующими в отсутствие поля (спонтанная поляризация), под действием поля величина пирокоэффициента испытывает скачки на границах фаз и аномальное увеличение при исчезновении или переключении компонент поляризации.

Наиболее интересное поведение пирокоэффициента наблюдается у пленок с деформациями, находящимися между точками $T_{1}$ и $T_{2}$ в области парафазы. Здесь пироэлектрический коэффициент под действием поля увеличивается от нуля до некоторого максимального значения, после чего начинает уменьшаться. Величина максимума зависит от значения вынужденной деформации и увеличивается по мере приближения к точке $T_{2}$.

Таким образом, управляемый электрическим полем пироэлектрический эффект существует для пленки BST07 в той области фазовой диаграммы, где отсутствует спонтанная поляризация (рис. 2). Величина экстре- мального значения пирокоэффициента, как видно из (5), неограниченно возрастает при приближении к точке потери устойчивости $t=1$. Для BST07 параметром $t$ является вынужденная деформация $u_{m}$, а точкой потери устойчивости - точка $T_{2}$ на рис. 2.

Для определения состава пленки твердого раствоpa BST, обладающей оптимальным значением пироэлектрического коэффициента, необходимо воспользоваться фазовой диаграммой вынужденная деформацияконцентрация, приведенной в работе [14]. Для заданного значения величины вынужденной деформации $u_{m}$ (заданы подложка, режим напыления и толщина пленки) на фазовой диаграмме следует определить точку на линии фазового перехода между парафазой и $a a$-фазой. Эта точка даст нужную концентрацию пленки BST.

Рассмотренная фазовая диаграмма вынужденная деформация-электрическое поле может быть полезна для практического применения, так как возможность изменения деформации в пленках позволяет осуществлять оптимизацию функции управления пироэлектрическим эффектом. Варьируя локальные напряжения в эпитаксиальных пленках BST путем изменения состава, можно достичь улучшения пироэлектрических свойств. В результате мы показали, что величина пироэлектрического коэффициента в пленке BST07, находящейся в режиме диэлектрического болометра, близка к наблюдаемой в типичных пироэлектрических материалах (например, в $\mathrm{PbZr}_{x} \mathrm{Ti}_{1-x} \mathrm{O}_{3}$ и других свинецсодержащих сегнетоэлектриках). Таким образом, можно получить высокий пироэлектрический отклик в бессвинцовых материалах и тем самым расширить спектр функциональных материалов, используемых в ИК-устройствах с требуемыми свойствами. 


\section{Список литературы}

[1] J.H. Haeni, P. Irvin, W. Chang, R. Uecker, P. Reiche, Y.L. Li, S. Choudhury, W. Tian, M.E. Hawley, B. Craigo, A.K. Tagantsev, X.Q. Pan, S.K. Streiffer, L.Q. Chen, S.W. Kirchoefer, J. Levy, D.G. Schlom. Nature 430, 758 (2004).

[2] K.J. Choi, M. Biegalski, Y.L. Li, A. Sharan, J. Schubert, R. Uecker, P. Reiche, Y.B. Chen, X.Q. Pan, V. Gopalan, L.-Q. Chen, D.G. Schlom, C.B. Eom. Science 306, 1005 (2004).

[3] A.K. Tagantsev, V.O. Sherman, K.F. Astafiev, J. Venkatesh, N. Setter. J. Electroceram. 11, 5 (2003).

[4] S. Gevorgian. Ferroelectrics in microwave devices, circuits and systems. Springer, London (2009). 396 p.

[5] R.W. Whatmore. Rep. Prog. Phys. 49, 1335 (1986).

[6] R.W. Whatmore. Ferroelectrics 118, 241 (1991).

[7] P. Muralt. Rep. Prog. Phys. 64, 1339 (2001).

[8] A. Rogalski. Prog. Quant. Electron. 27, 59 (2003).

[9] R.W. Whatmore, Q. Zhang, C.P. Shaw, R.A. Dorey, J.R. Alcock. Phys. Scripta T 129, 6 (2007).

[10] M. Dawber, K.M. Rabe, J.F. Scott. Rev. Mod. Phys. 77, 1083 (2005).

[11] J.F. Scott. Science 315, 954 (2007).

[12] B.W. Wessels. Ann. Rev. Mater. Res. 37, 659 (2007).

[13] V.B. Shirokov, V.I. Torgashev, A.A. Bakirov, V.V. Lemanov. Phys. Rev. B 73, 104116 (2006).

[14] V.B. Shirokov, Yu.I. Yuzyuk, B. Dkhil, V.V. Lemanov. Phys. Rev. B 79, 144118 (2009).

[15] J.F. Nye. Physical properties of crystals. Clarendon Press, Oxford (1964). 352 p.

[16] R. Watton. Ferroelectrics 91, 87 (1989). 